\title{
Conhecimento dos profissionais da rede pública de saúde sobre Constelação Familiar
}

\author{
Knowledge of public health professionals about Family Constellation \\ Conocimiento de los profesionales de la salud pública sobre la Constelación Familiar \\ Beatriz Ritzmann Ferraz' ${ }^{1}$ Lívia Oliveira Rosa' ${ }^{1}$, Camila Gonçalo-Mialhe ${ }^{1 *}$.
}

\begin{abstract}
RESUMO
Objetivo: Verificar o conhecimento dos profissionais da rede pública de saúde sobre Constelação Familiar. Métodos: Estudo exploratório quantiqualitativo realizado por meio de questionários eletrônicos aplicados aos profissionais que atuam na rede pública em unidades de saúde que of erecem e que não of erecem Práticas Integrativas e Complementares em Saúde (PICS). As respostas foram analisadas tematicamente nas questões abertas e por meio de estatística descritiva nas questões fechadas. Resultados: Dos 20 participantes, 16 declararam sexo feminino (80\%); 11 mencionaram saber o que era a CF $(55 \%)$; $16(80 \%)$ desconhecem que a CF é oferecida pelo SUS desde 2018. Os respondentes apresentaram "ideias convergentes" e "ideias divergentes" em relação ao conceito de CF adotado pelo Ministério da Saúde e manifestaram que a CF poderia contribuir com a área da saúde auxiliando os usuários a lidarem com os sentimentos/emoções e auxiliando os usuários a lidarem com as doenças. Conclusão: Embora a CF seja uma PIC reconhecida e seu uso na rede pública de saúde esteja regulamentado, os participantes desta pesquisa apresentaram conhecimentos incipientes sobre o tema.
\end{abstract}

Palavras-chave: Saúde mental, Atenção primária à saúde, Sistema único de saúde.

\begin{abstract}
Objective: To verify the knowledge of public health professionals about the family constellation. Methods: The transversal, quantitative and qualitative study was made by electronic questionnaires applied to professionals who work in the public health system in both units that offer or do not offer appointm ents of Integrative and Complementary Practices in Health (ICPH). The opened answers were analysed by theme as opposed to the closed ones, that were analysed by descriptive statistics. Results: Among the 20 participants, 16 were declared females (80\%) and 11 (55\%) declared knowing what FC is; $16(80 \%)$ don't know that FC has been offered by SUS since 2018. The participants had "converging" and "diverging" ideas about the FC's concept adopted by the Health Ministry and manifested that FC could contribute in the health field "helping users to deal with their feelings/emotions" and "assisting the users to deal with disease". Conclusion: Although FC is a known and recognized ICP and its use in the public health system is established, the participants of this research presented insufficient knowledge regarding the theme.
\end{abstract}

Key words: Mental health assistance, Primary health care, Unified health system.

\section{RESUMEN}

Objetivo: Verificar el conocimiento de los profesionales de la salud pública sobre la constelación familiar. Métodos: Estudio exploratorio cuantitativo y cualitativo realizado mediante cuestionarios electrónicos aplicados a profesionales que laboran en la red pública en unidades de salud que ofrecen y no ofrecen Prácticas Integrativas y Complementarias en Salud (PICS). Las respuestas se analizaron temáticamente en

${ }^{1}$ Faculdade de Medicina de Jundiaí (FMJ), Jundiaí - SP. *E-mail: camilagoncalo@g.fmj.br

SUBMETIDO EM: 12/2021

ACEITO EM: 12/2021

PUBLICADO EM: 12/2021 
las preguntas abiertas y mediante estadística descriptiva en las preguntas cerradas. Resultados: De los 20 participantes, 16 indicaron sexo femenino (80\%); 11 afirmaron conocer lo que era CF (55\%); 16 (80\%) desconocen que él SUS ofrece CF desde 2018. Los encuestados presentaron "ideas convergentes" e "ideas divergentes" en relación al concepto de CF adoptado porel Ministerio de Salud y expresaron que el CF podría contribuir al área de salud "ayudar a los usuarios a lidiar con sus sentimientos/ emociones" y "ayudar a los usuarios a lidiar con las enfermedades". Conclusión: Aunque CF es una PIC reconocida y su uso en la red de salud pública está regulado, los participantes de esta investigación mostraron un conocimiento incipiente sobre el tema.

Palabras clave: Atención a la salud mental, Atención primaria de salud, Sistema único de salud.

\section{INTRODUÇÃO}

A Constelação Familiar (CF) é uma abordagem terapêutica que foi sistematizada pelo alemão Bert Hellinger. Os fundamentos da CF abrangem questões humanísticas, filosóficas e antropológicas. As Constelações Sistêmicas consideram "sistema" como algo composto por mais de um indivíduo e pelas interações mútuas que acontecem neste cenário. Elas englobam a Constelação Familiar, a Pedagogia Sistêmica, o Direito Sistêmico, a Constelação Organizacional e também a Saúde Sistêmica (FRANCELINO ET, et al., 2018).

No Brasil a abordagem sistêmica tem sido utilizada no judiciário (Direito Sistêmico), na área da educação (Educação Sistêmica ou Pedagogia Sistêmica), na área empresarial (Constelação Organizacional), na área da saúde privada e pública, sendo que nesta última a CF é uma das 29 modalidades de Práticas Integrativas e Complementares em Saúde (PICS) incluídas no Sistema Único de Saúde (SUS) (SOUZA TT, 2021; BERNÁRDEZ-GÓMEZ A e BELMONTE ML, 2021; BARRO NB, 2019; MINISTÉRIO DA SAÚDE, 2018).

No âmbito do direito, o emprego da abordagem sistêmica tem gerado impacto positivo. A literatura da área menciona que a prática do modelo sistêmico neste contexto gera mudança de postura entre as partes envolvidas nos processos, imprimindo uma nova dinâmica, deixando posicionamentos litigantes, assumindo atitudes consensuais, resultando na procura de soluções para os envolvidos na questão judicial. Estas conquistas contribuem com o aumento da agilidade na resolução dos processos, fato que auxilia na redução do volume das ações judiciais acumuladas e agiliza o ritmo de finalização delas (VALL J, 2018).

$\mathrm{Na}$ área da educação, há uma publicação referente ao emprego da CF como instrumento pedagógico e de mediação entre a escola e a família, cujos resultados promoveram benefícios no comportamento em sala de aula, na redução de conflitos envolvendo alunos e professores, bem como no aumento do interesse pelo aprendizado (FRANCELINO ET, et al., 2017).

O Glossário temático: práticas integrativas e complementares em saúde, publicado pelo Ministério da Saúde (2018) define CF como método de ordem psicoterapêutica que of erece um olhar sistêmico, energético e fenomenológico utilizado para identificar o ponto de partida de questões manifestadas pelo usuário e/ou situações conflituosas que atingem sua vida familiar. Ao identificar os componentes que estão agindo no inconsciente familiar e aplicando as leis sistêmicas da ordem, do equilíbrio e do pertencimento, é possível que o usuário manifeste mudanças na sua trajetória pela busca de seu crescimento pessoal e de sua cura .

Até o momento, as publicações sobre CF na área da saúde são escassas. Entretanto, há pesquisas que verificaram contribuições desta abordagem terapêutica para a saúde física e mental. Como exemplo, tem-se o estudo que constatou a influência da CF em casos de pacientes com dermatite atópica/psoríase e a pesquisa que explorou as contribuições da CF combinada com outras intervenções em quadros de indivíduos com depressão (JAFFERANY M, et al., 2019; RAMOS S e RAMOS J, 2019).

Considerando a existência da regulamentação para implantação da CF no sistema de saúde pública of erecido no Brasil e que este é um assunto pouco explorado na literatura, o objetivo desta pesquisa foi verificar o conhecimento sobre a CF entre profissionais que atuam do SUS. 


\section{MÉTODOS}

Trata-se- de um estudo exploratório fruto de uma iniciação científica realizada no período de 2019 a 2020. A aprovação pelo Comitê de Ética se deu em 12/09/2019 (CAEE: 15375619.7.0000.5412; Número do Parecer: 3.553.3330). Inicialmente, foi necessário localizar as informações das unidades de saúde e dos profissionais que atuam nestes serviços. Assim, as pesquisadoras entraram em contato com gerentes de unidades de saúde e fizeram a verificação dos locais que estariam disponíveis para a coleta dos dados.

Visando a constatação da clareza e veracidade das perguntas e respostas, foi feito um pré-teste do questionário durante o mês de novembro de 2019, observando a necessidade de correções de possíveis distorções e problemas relacionados com as perguntas. Após os devidos ajustes neste instrumento, foram agendados os horários para a obtenção dos dados, procedimento que se deu entre os meses de abril e julho de 2020. Ressalta-se que a ideia original era realizar pessoalmente a aplicação dos questionários, entretanto, considerando o cenário da pandemia COVID-19, a captação das respostas foi realizada mediante preenchimento de um formulário eletrônico.

Foram convidados profissionais que atuam na rede pública de saúde em unidades que of erecem Práticas Integrativas e Complementares em Saúde (PICS) e aqueles que trabalham em unidades que não oferecem essas práticas. Todas as respostas foram organizadas em tabelas. Informações numéricas passaram por análise quantitativa via estatística descritiva. A coleta dos dados foi encerrada quando se constatou a saturação teórica das respostas (FONTANELLA BJB, et al., 2008). Todos os voluntários assinaram o Termo de Consentimento Livre e Esclarecido (TCLE). Visando manter o sigilo ético dos dados, cada indivíduo recebeu uma codificação, utilizando a letra $\mathrm{F}$ e o número referente a sequência de participação na coleta de dados.

O material proveniente das questões abertas foi analisado qualitativamente por meio de eixos temáticos. Realizou-se a leitura e releitura de todas as respostas com a finalidade de identificar um conjunto inicial de temas ou categorias (busca de ideias recorrentes e itens de interesse, como por exemplo: pontos de vista incomuns, tipos particulares de narrativas).

Em seguida, foram criadas categorias que possibilitaram uma reflexão sobre as nuances dos discursos. A realização desta etapa permitiu agrupar e conectar itens dos dados que contemplavam mais de uma categoria. Após o refinamento das categorias estabelecidas, foi possível identificar os temas. Esta identificação permitiu a estruturação do material em tabelas, facilitando a conferência da inclusão e comparação dos temas agrupados, perfazendo a análise temática.

A etapa final desta análise se deu por meio da redação de uma síntese interpretativa que buscou criar um diálogo entre termos e objetivos, questões e pressupostos do presente estudo (POPE C e MAYS N, 2019; GOMES R, 2016).

\section{RESULTADOS E DISCUSSÃO}

Foram preenchidos 20 questionários eletrônicos, sendo 10 desses completados por voluntários que trabalham em uma unidade de saúde que oferece PIC (UCPIC: Unidade de Saúde Com PIC) e 10 voluntários que atuam em uma unidade de saúde que não oferece PIC (USPIC: Unidade de Saúde Sem PIC). Os 10 participantes da UCPIC são maioria do sexo feminino $(n=08 ; 80 \%)$, e exercem os cargos de: Médicas $(n=03$, $30 \%$ ); Técnicas de Enfermagem ( $n=02 ; 20 \%)$; Agente de Saúde ( $n=02 ; 20 \%)$; Assistente Administrativo ( $n=01$; $10 \%)$; Cirurgião Dentista $(n=01 ; 10 \%)$ e Enfermeira $(n=01 ; 10 \%)$. Na USPIC, os 10 respondentes também são majoritariamente do sexo feminino $(n=08 ; 80 \%)$, exercendo os cargos de Técnica em Enfermagem ( $n=06$; $60 \%)$, Gerência ( $n=01 ; 10 \%)$; Enfermeira $(n=01 ; 10 \%)$; Agente de Saúde $(n=01 ; 10 \%)$; Auxiliar de Enfermagem $(\mathrm{n}=01 ; 10 \%)$.

A questão 01 tratava do conhecimento sobre Constelação Familiar (CF) e a questão 02 versava a respeito do conhecimento da of erta de CF no SUS. As respostas referentes à essas perguntas estão organizadas na Tabela 1. 
Tabela 1 - Dados referentes às respostas das questões 02 e 03.

\begin{tabular}{|c|c|c|c|c|}
\hline \multicolumn{5}{|c|}{ Questão 01 - O/A Sr(a) sabe o que é Constelação Familiar? } \\
\hline \multirow{2}{*}{ Unidade de Saúde } & \multicolumn{2}{|c|}{ Sim } & \multicolumn{2}{|c|}{ Não } \\
\hline & $\mathbf{n}$ & $\%$ & $\mathbf{n}$ & $\%$ \\
\hline USPIC & 6 & 30 & 4 & 20 \\
\hline UCPIC & 5 & 25 & 5 & 25 \\
\hline Total & 11 & 55 & 9 & 45 \\
\hline \multicolumn{5}{|c|}{ Questão 02 - O/A Sr (a) sabe que a Constelação Familiar é oferecida pelo SUS desde 2018? } \\
\hline \multirow{2}{*}{ Unidade de Saúde } & \multicolumn{2}{|c|}{ Sim } & \multicolumn{2}{|c|}{ Não } \\
\hline & $\mathbf{n}$ & $\%$ & $\mathbf{n}$ & $\%$ \\
\hline USPIC & 3 & 15 & 7 & 35 \\
\hline UCPIC & 1 & 5 & 9 & 45 \\
\hline Total & 4 & 20 & 16 & 80 \\
\hline
\end{tabular}

Fonte: Ferraz BH, et al., 2021.

Embora a maioria dos participantes tenha declarado conhecer a CF, poucos sabiam do reconhecimento e da recomendação desta PIC na rede pública de saúde. Este achado faz um convite à reflexão sobre o papel do Brasil como referência mundial no uso das PICS na Atenção Básica e sobre a questão da formação de recursos humanos em saúde que pouco aborda este assunto durante a graduação dos profissionais (SANTOS DS, et al, 2014; COELHO MTAD, et al., 2019; BATALHA E, 2020). Neste sentido, torna-se importante recuperarmos a noção da educação permanente, que necessita estar atrelada com as bases da atenção, gestão e educação em saúde para que a disseminação do conhecimento atualizado entre os trabalhadores da área aconteça, minimizando o impacto da falta de conhecimento como principal origem das barreiras de acesso. O Gráfico 1 apresenta os valores absolutos $(n)$ e relativos (\%) referentes às respostas da questão 03: O/A Sr (a) imagina o que seria a Constelação Familiar? Se sim, por favor, descreva/comente.

Gráfico 1 - Valores absolutos (n) e relativos (\%) referentes às respostas da questão 03.

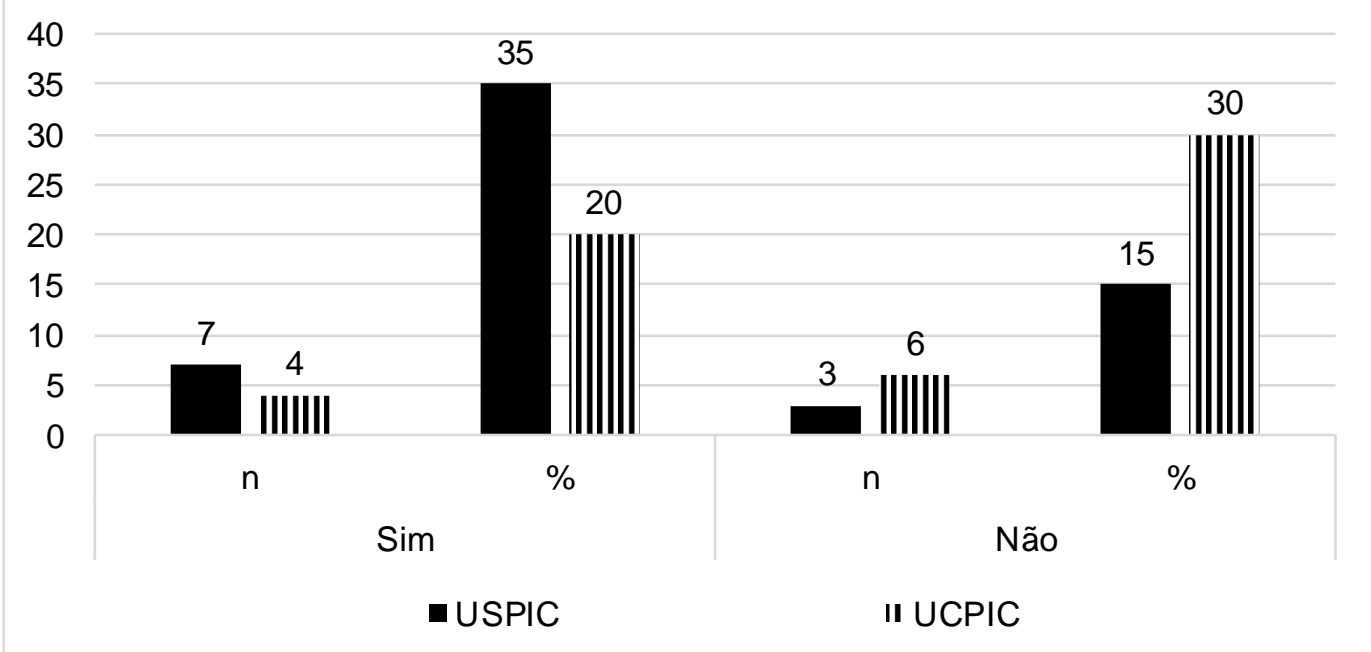

Fonte: Ferraz BH, et al., 2021.

Os achados qualitativos registrados nas 11 respostas que descreveram o que seria a $C F$, foram organizados em eixos temáticos, embasados pela definição de CF descrita no Glossário Temático - Práticas Integrativas e Complementares em Saúde (MINISTÉRIO DA SAÚDE, 2018). Assim foi possível identificar que os participantes da pesquisa, ao descreverem o que imaginavam ser a $\mathrm{CF}$, apresentaram ideias que se aproximavam e se distanciavam do conceito de CF utilizado no glossário. Esse contraste pode ser reconhecido nos extratos que seguem:

"É um método psicoterapêutico que estuda os padrões de comportamento de grupos familiares através de suas gerações" (F5, Feminino, Técnica Enfermagem, USPIC). 
Pode-se observar a consonância da resposta acima com a definição de CF no referido glossário:

"Método psicoterapêutico de abordagem sistêmica, energética e fenomenológica, que busca reconhecer a origem dos problemas e/ou alterações trazidas pelo usuário, bem como o que está encoberto nas relações familiares para, por meio do conhecimento das forças que atuam no inconsciente familiar e das leis do relacionamento humano, encontrar a ordem, o pertencimento e o equilíbrio, criando condições para que a pessoa reoriente o seu movimento em direção à cura e ao crescimento" (MINISTÉRIO DA SAÚDE, 2018).

Outro fato que pode ser ressaltado referente a esta resposta, é que a profissional de saúde atua em uma unidade que não oferece PICS, entretanto, demonstrou conhecimento sobre o assunto. Abaixo segue exemplo de uma resposta que se distancia do conceito de CF utilizado pelo Ministério da Saúde:

"Aconselhamento familiar para que sejam resolvidos conflitos da melhor forma possível' (F6, Feminino, Técnica de Enfermagem, USPIC).

Ressalta-se que CF não opera na lógica do aconselhamento. A CF coloca o indivíduo em evidência, expondo características da questão abordada na sessão, visando ajudá-lo no reconhecimento e na mudança da posição em que ele se sente atado a uma dificuldade que se repete com frequência em sua vida (HELLINGER B, 2019). Portanto, a CF viabiliza a possibilidade de transformação dos indivíduos, pois, revela os enredamentos que originam conflitos que parecem insolúveis dentro do sistema. Ao mesmo tempo, há a exposição das soluções que são aplicadas durante a sessão.

A literatura sobre CF explica que no decorrer da realização de uma CF a pessoa atendida se conecta com um cenário composto de informações provenientes da sua consciência individual e de uma consciência maior. É nesse cenário que o sujeito se movimenta, e, por meio desta movimentação, cria um novo panorama que passa a atuar dentro do sistema. Assim é concretizada a transformação necessária, trazendo um olhar atual para o cenário em questão. Deste modo, a intervenção terapêutica consiste basicamente na revelação da dinâmica do sistema, partindo de uma visão inicial limitada e chegando, ao final da sessão, a um panorama ampliado, organizado e resolvido (BRAGA ALA, 2009).

Na questão 04, a pergunta era: O Sr/A Sr (a) imagina como a Constelação Familiar poderia ajudar na área da saúde? Se sim, por favor, descreva/comente. Os resultados quantitativos apontam que $55 \%$ ( $n=11)$ dos respondentes imaginam como a CF poderia ajudar na área da saúde. Esses dados estão detalhados no

\section{Gráfico 2.}

Gráfico 2 - Valores absolutos (n) e relativos (\%) referentes às respostas da questão 04.

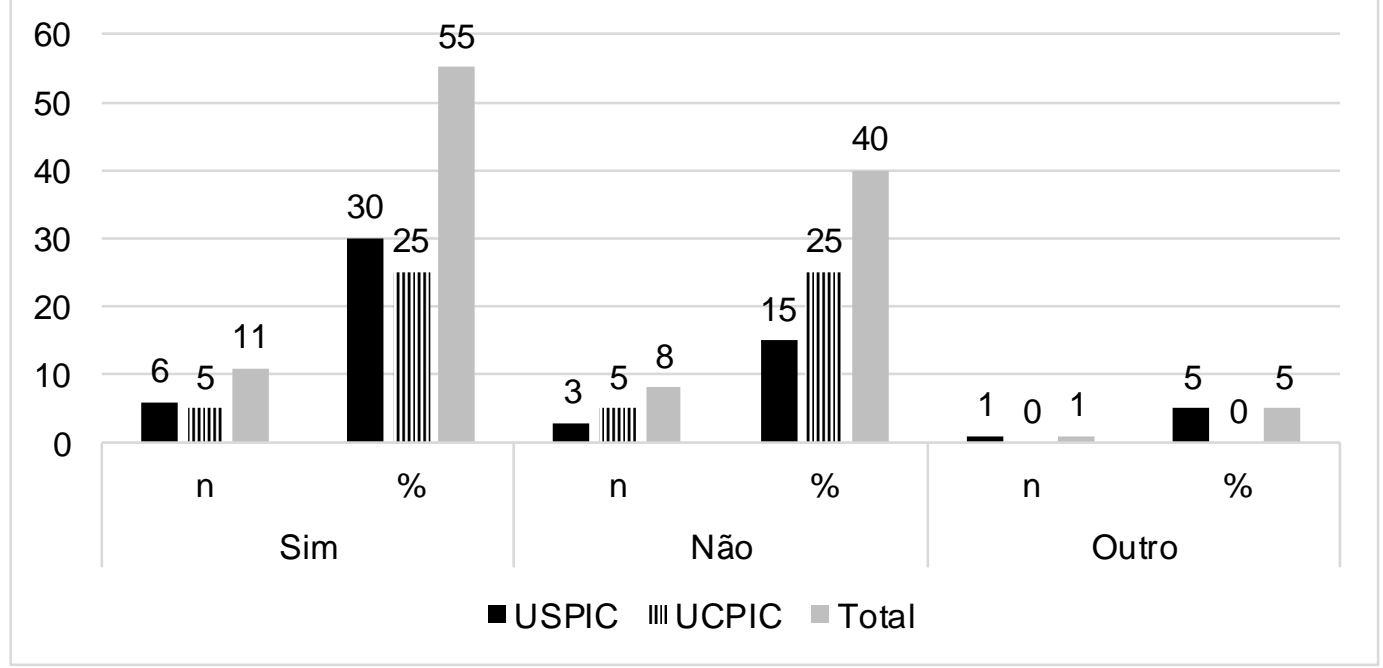

Fonte: Ferraz BH, et al., 2021. 
A análise qualitativa da descrição dos participantes de como a CF poderia ajudar na área da saúde, resultou em dois eixos temáticos principais: 1) auxiliando os usuários a lidarem com os sentimentos/emoções; 2) auxiliando os usuários a lidarem com as doenças. Abaixo seguem respostas que representam o primeiro eixo e a respectiva discussão.

"Atender as famílias que atravessam dificuldades emocionais" F5, Feminino, Técnica Enfermagem, USPIC).

"Acho que poderia ajudar no sentido de autoconhecimento dos usuários" (F12, Feminino, Técnica Enfermagem, UCPIC).

Os sujeitos que responderam os questionários, atuam no contexto da APS, portanto, tem suas práticas alicerçadas no enfoque comunitário e familiar, buscando atender ao máximo as necessidades em saúde da população sob seus cuidados (HEIMANN LS, et al., 2011).

Assim, os trechos citados ilustram o comprometimento dos respondentes com seu campo de atuação, preocupando-se com fatores que extrapolam o componente biológico das doenças. Nessa conjuntura, a parte emocional/sentimental e de autoconhecimento foi destacada pelos respondentes ao externarem suas ideias sobre como a CF poderia ajudar na área da saúde. Alguns autores buscaram identificar o significado atribuído pelos usuários à saúde, à doença e à unidade de saúde. Eles verificaram que tais indivíduos encaram o autocuidado como elemento determinante para conservação da saúde, veem a doença como incapacidade ou déficit para o autocuidado e colocam que o serviço de saúde significa a recuperação da saúde e da capacidade para o autocuidado (SANTOS DS, et al., 2014).

As influências de "emoções negativas" e "emoções positivas" geram reflexos no organismo e imprimem somatização no corpo físico, configurando o cenário de doença ou de saúde. Assim, quando o indivíduo é auxiliado e estimulado ao desenvolvimento de "emoções positivas", tem-se repercussão na melhoria da sua qualidade de vida (GIMENEZ RM e BERVIQUE JA, 2006). Outra vertente, a Psiconeuroimunologia, se dedica ao estudo das relações entre fatores estressores psicossociais, as emoções e o sistema imunológico que estrutura a resposta adaptativa ao estresse. Esta linha de estudo, considera que os estressores psicossociais reduzem a eficiência do sistema imunológico, promovendo, então, o aumento de sintomas médicos (MAIA A C, 2002).

Alguns pesquisadores def endem a ideia de que o componente emocional pode ser responsável por várias doenças que afetam o corpo (ANDRADE A, et al., 2005; GOUVEIA EC e ÁVILA LA, 2010; CRUZ MZ e PEREIRA JRA, 2011). Neste sentido, quando o indivíduo se coloca em uma sessão de CF e ressignifica os entrelaçamentos que o bloqueavam, ele se põe como protagonista, trabalhando seu autoconhecimento, 0 gerenciamento de suas emoções/sentimentos, podendo contribuir com a melhoria da própria qualidade de vida. Aqui são apresentadas respostas que representam o segundo eixo e a respectiva discussão:

"No meu ver, ajudaria muito com pacientes com doenças crônicas, que tem dificuldade em aceitar o tratamento, e seus familiares" (F8, Feminino, Agente de Saúde, USPIC).

"Melhor compreensão do significado da doença para o indivíduo" (F17, Feminino, Médica, UCPIC).

É importante ajudar indivíduos doentes a alcançarem a superação das dificuldades relacionadas a suas doenças. O adoecimento crônico, principalmente quando afeta o corpo físico, gera muitas restrições, despertando temores e revelando limites na autonomia do indivíduo acometido. Neste sentido, é importante lançar mão de um recurso que auxilie o indivíduo e seus familiares a reconhecerem as limitações impostas pelas doenças crônicas, bem como os ajude na superação das dificuldades que se apresentam (BIGHETI A e DO VALLE ERM, 2008).

O médico Stephen Hausner, em sua obra "Constelações Familiares e o Caminho da Cura - A abordagem da doença sob a perspectiva de uma medicina integral", comunica que é possível colocar a doença ou o 
sintoma como tema a ser trabalhado na sessão de CF. Esta colocação permite identificar com qual postura o indivíduo olha para a doença/sintoma e nesta dinâmica emergem as conexões que geralmente são inconscientes. No decorrer do esclarecimento da questão, o sujeito manifesta sua postura em relação a doença ou ao sintoma e mostra sua vontade de enfrentá-los, bem como seu estado de ânimo para se defrontar com fatos e situações que estão por trás do cenário vivenciado na sessão de CF. Hausner destaca que o trabalho com CF para pessoas doentes é uma via favorável, eficiente e que complementa o cuidado médico of erecido a esses indivíduos (HAUSNER S, 2010).

Nas questões 05, 06 e 07 a maior parte dos voluntários utilizou o termo "não sei" nas respostas sobre o caminho para se implantar a of erta de práticas integrativas e complementares em um município, bem como sobre o recebimento de alguma orientação proveniente de órgão público para iniciar a implantação do serviço de CF na unidade de saúde em que trabalha e sobre a previsão da oferta de CF na unidade de saúde em que atua. Os dados detalhados estão apresentados na Tabela 2.

Tabela 2 - Dados referentes às respostas das questões 05,06 e 07.

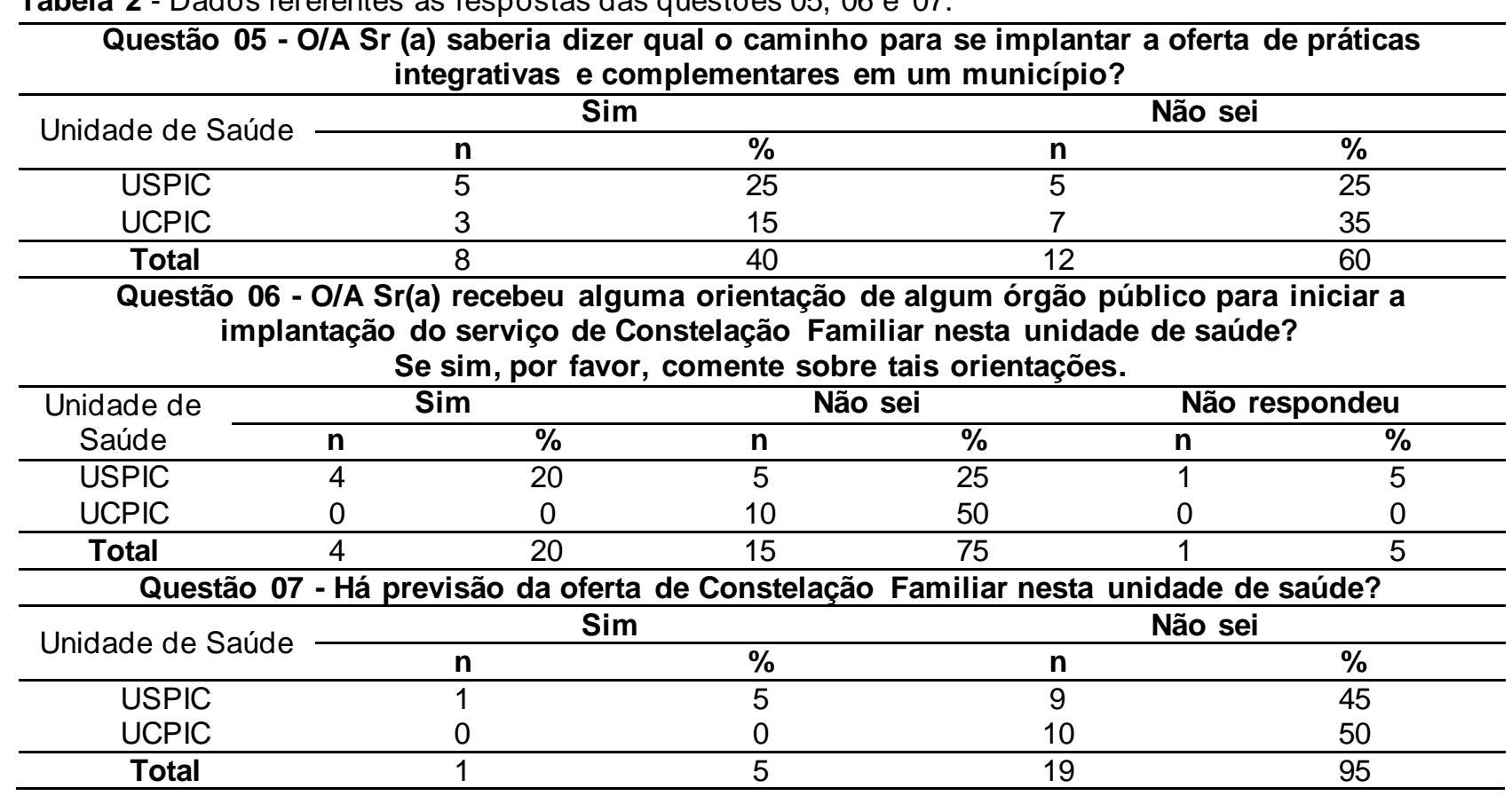

Fonte: Ferraz BH, et al., 2021.

Referente ao caminho a ser percorrido para implantar PICS em um município, a maior parte das respostas indicou desconhecimento sobre o assunto, entretanto, destaca-se aqui a existência de documentos oficiais sobre o tema. O "Manual de implantação de serviços de Práticas Integrativas e Complementares no SUS" aponta que este processo pode seguir duas grandes fases: a primeira, denominada "definição da proposta", que contempla o levantamento dos atores responsáveis, o diagnóstico situacional e a análise organ izacional. A segunda fase, é composta pela "elaboração do plano de desenvolvimento de implantação das PICS".

Esta segunda fase compreende a regulamentação da of erta das PICS; a capacitação profissional; o apoio matricial; a cooperação horizontal (compartilhamento de experiências exitosas que podem ser aplicadas na rotina de trabalho das equipes envolvidas); a criação de serviços na Atenção Básica; a criação de serviços de especialidades em PICS; a criação de serviços hospitalares e serviços ligados às redes temáticas; o cadastramento dos serviços de PICS no Cadastro Nacional de Estabelecimentos de Saúde (SCNES) e a divulgação do plano (MINISTÉRIO DA SAÚDE, 2018).

No âmbito municipal existem alguns documentos que mencionam a questão das PICS na rede pública de saúde. Por exemplo, o relatório final da 9ª Conferência Municipal de Saúde cita a implantação de uma unidade 
de PICS na assistência of erecida às unidades de saúde da rede municipal e relata que a implementação das PICS no SUS nesta cidade tem a finalidade de prevenir agravos à saúde e promover qualidade de vida.

Outros documentos que podem ser encontrados no portal eletrônico da prefeitura, reforçam e complementam o referencial para implementação das PICS no SUS. Inclusive, os dados referentes à Conferência Municipal de Saúde de 2017, revelam um "indicador para quantificar o acesso às PICS". O mesmo documento versa sobre o objetivo de "garantir que 100\% das Unidades de Atenção Básica of ereçam PICS". Nota-se que existe referencial teórico sobre a implementação de PICS tanto no âmbito municipal, quanto no âmbito federal, porém, este conhecimento parece não ter sido acessado pelos profissionais que participaram da presente pesquisa (MINISTÉRIO DA SAÚDE, 2018; SECRETARIA DE SAÚDE, 2011; SECRETARIA DE SAÚDE, 2017; PREFEITURA MUNICIPAL DE JUNDIAÍ, 2018).

\section{CONCLUSÃO}

Publicações recentes sobre Constelação Familiar (CF) no SUS são escassas. Neste sentido, este artigo é uma contribuição para a área das PICS na Atenção Primária em saúde. Considerando o objetivo do estudo e os resultados das análises realizadas, conclui-se que embora a CF seja uma PICS reconhecida e seu uso na rede pública de saúde esteja regulamentado, os participantes desta pesquisa apresentaram conhecimentos incipientes sobre o tema. As ideias sobre CF manifestadas pelos respondentes apontaram convergências e divergências com o conceito de CF adotado pelo Ministério da Saúde, bem como indicam que esta PICS pode auxiliar os usuários a lidarem com sentimentos, emoções e doenças.

\section{AGRADECIMENTOS E FINANCIAMENTO}

Agradecemos o apoio da Unidade de Gestão de Promoção da Saúde do município de Jundiaí e a bolsa de iniciação científica of erecida pelo PIBIC/FMJ, processo número: 2544067016.

\section{REFERÊNCIAS}

1. ANDRADE A et al. Aspectos emocionais e psicossomáticos no processo de recuperação psicomotora. Revista Digital, 2005; (84): 1-1.

2. BARRO NB. Uma visão sistêmica sobre o assédio moral no âmbito do trabalho. Rev. do Trib. Reg. Trab. 10ª Região, 2019;23 (2): 105-110.

3. BATALHA E. Saúde em várias dimensões. Revista Radis ENSP, 2020; (210): 22-29.

4. BERNÁRDEZ-GÓMEZ A, BELMONTE ML. Pedagogia sistêmica e desenvolvimento pessoal na educação, questões a serem consideradas. Brazilian Applied Science Review, 2021;5 (1):69-85.

5. BIGHETTI A, DO VALLE ERM. Compreendendo as vivências de adolescentes com câncer: análise fenomenológica do TAT. Aletheia, 2009; (30): 88-100.

6. BRAGA ALA. Psicopedagogia e constelação familiar sistêmica: um estudo de caso. Rev. psicopedag ., 2009;26(80): 274-285.

7. COELHO MTAD, et al. Representações sociais de doença, usos e significados atribuídos às Práticas Integrativas e Complementares por universitários. Saúde debate, 2019;43 (122):848-862.

8. CRUZ MZ, PEREIRA JRA. Corpo, mente e emoções: Referenciais Teóricos da Psicossomática. Rev. Simbio-Logias, 2011; 4 (6): 46-65.

9. FONTANELLA BJB et al. Amostragem por saturação em pesquisas qualitativas em saúde: contribuições teóricas. Cad. Saúde Pública, 2008;24 (1): 17-27.

10. FRANCELINO EF et al. A Constelação Familiar Sistêmica como uma Ferramenta Pedagógica e de Mediação entre Família e Escola. R Est Inv Psico y Educ, 2017; Extr.(5): A5-340.

11. FRANCELINO EF et al. O impacto da Constelação Familiar Sistêmica na saúde emocional dos discentes da EEFM João Mattos. Anais do IV COLBEDUCA - Colóquio Luso-Brasileiro de Educação, 2018; (3): 1-18.

12. GIMENEZ RM, BERVIQUE JA. Relações entre as emoções e o organismo como um todo. Revista Científica Eletrônica de Psicologia, 2006; (7): 1-6.

13. GOMES R. Análise e interpretação de dados de pesquisa qualitativa. In: Minayo MCS (org.). Pesquisa social:Teoria, método e criatividade - Série Manuais Acadêmicos. Petrópolis: Vozes, 2016;96p.

14. GOUVEIA EC, ÁVILA LA. Aspectos emocionais associados a disfunções gastroenterológicas. Psicologia em Estudo, 2010; 15 (2): 265-273.

15. HAUSNER S. Constelações Familiares e o Caminho da Cura. A abordagem da doença sob a perspectiva de uma medicina integral. São Paulo: Cultrix, 2010; 240p. 
16. HEIMANN LS et al. Atenção primária em saúde: um estudo multidimensional sobre os desafios e potencialidades na Região Metropolitana de São Paulo (SP, Brasil). Ciência \& Saúde Coletiva, 2011;16 (6): 2877-2887.

17. HELLINGER B. As ordens do amor. Um Guia Para o Trabalho com Constelações Familiares. São Paulo: Cultrix, 2019;424p.

18. JAFFERANY M, et al. Effects of family constellation seminars on itch in patients with atopic dermatitis and psoriasis: A patient preference controlled trial. Dermatologic Therapy, 2019;32(6):e13100.

19. MAIA AC. Emoções e sistema imunológico:um olhar sobre a Psiconeuroimunologia. Psicologia: Teoria, Investigação e Prática, 2002; (2): 207-225.

20. MINISTÉRIO DA SAÚDE. Portaria $n^{\circ}$ 702, de 21 de março de 2018. Altera a Portaria de Consolidação nº 2/GM/MS, de 28 de setembro de 2017, para incluir novas práticas na Política Nacional de Práticas Integrativas e Complementares (PNPIC). Disponível em: https://bvsms.saude.gov.br/bvs/saudelegis/gm/2018/prt0702_22_03_2018.html. Acessado:3 de dez. de 2021.

21. MINISTÉRIO DA SAÚDE. Glossário temático: práticas integrativas e complementares em saúde. Dispon ível em: https://portalarquivos2.saude.gov.br/images/pdf/2018/marco/12/glossario-tematico.pdf. Acessado:3 de dez. de 2021.

22. MINISTÉRIO DA SAÚDE. Manual de implantação de serviços de práticas integrativas e complementares no SUS. Disponível em: http://189.28.128.100/dab/docs/portaldab/publicacoes/manual_implantacao_servicos_pics.pdf. Acessado: 3 de dez. de 2021.

23. PREFEITURA MUNICIPAL DE JUNDIAÍ. Plano Municipal de Saúde:2018 - 2021. Aprovado pelo Conselho Municipal de Saúde em:30.08.2018. Disponívelem:https://jundiai.sp.gov.br/saude/wp-content/uploads/sites/17/2017/09/planomunicipal-de-saude-jundiai-2018-2021.pdf. Acessado: 3 de dez. de 2021.

24. POPE C, MAYS N. Qualitative Research in Health Care. Hoboken: John Wiley \& Sons Ltd., 2019; 249p.

25. RAMOS S, RAMOS J. Process of Change and Effectiveness of Family Constellations: A Mixed Methods Single Case Study on Depression. The Family Journal, 2019;27(4): 418-428.

26. SANTOS DS et al. Processo saúde/doença e estratégia de saúde da família: o olhar do usuário. Rev. Latino-Am. Rev. Latino-Am. Enfermagem, 2014;22 (6): 918-25.

27. SECRETARIA DE SAÚDE. Conselho Municipal de Saúde - Jundiaí, Prefeitura Municipal de Jundiaí. Relatório Final da $9^{a}$ Conferência Municipal de Saúde de Jundiaí: 2 de julho de 2011. 2011. Disponível em: https://jundiai.sp.gov.br/saude/wp-content/uploads/sites/17/2014/08/rf9c.pdf. Acessado:3 de dez. de 2021.

28. SECRETARIA DE SAÚDE. Conselho Municipal de Saúde - Jundiaí, Prefeitura Municipal de Jundiaí. Ata da Conferência Municipal de Saúde de Jundiaí: 13 de julho de 2017. 2017. Disponível em: https://jundiai.sp.gov.br/saude/wp-content/uploads/sites/17/2017/07/ATA-e-relatorio-final-da-conferencia-municipalde-saude-2017.pdf. Acessado: 3 de dez. de 2021.

29. SOUZA TT. Direito Sistêmico uma análise da Constelação Familiar como instrumento sistêmico de humanização do Direito. São Paulo: Editora Dialética, 2021;108p.

30. VALL J. Direito Sistêmico: o Modelo de Constelação de Bert Hellinger e a Teoria da Complexidade de Edgar Morin convergências e significâncias. Anais do XIII Encontro de Iniciação Científica do Centro Universitário 7 de Setembro, 2017;7(1): 1-18. 